9th A. Friedmann International Seminar and

3rd Casimir Symposium 2015

International Journal of Modern Physics: Conference Series

Vol. 41 (2016) 1660130 (9 pages)

(C) The Author(s)

DOI: $10.1142 /$ S2010194516601307

\title{
Neutron and quark stars in $f(R)$ gravity
}

\author{
Artyom V. Astashenok \\ Institute of Physics and Technology, I. Kant Baltic Federal University, \\ Nevskogo 14, Kaliningrad, 236041, Russia \\ artyom.art@gmail.com
}

Received 28 August 2015

Revised 15 September 2015

Published 18 March 2016

\begin{abstract}
The realistic compact star models are considered in $f(R)$ gravity. The main result is that simple modified gravity can be consistent with current observational mass limit of neutron stars, with current data on their masses and radii; also, it simplifies solution of the hyperon problem. The effect of strong magnetic field in $R^{2}$-gravity is also investigated. The existence of more compact (in comparison with General Relativity) stars with large magnetic fields in central regions is possible. In fact the second branch of stability appears.
\end{abstract}

Keywords: Modified gravity; neutron stars; strange stars.

PACS numbers: 97.60.Jd, 04.50.Kd

\section{Introduction}

Recent observations lead to conclusion that our Universe expands with acceleration. ${ }^{1-3}$ In frames of General Relativity one needs to include into consideration the so called "dark energy" for explaining this phenomena. The dark energy can be viewed as some cosmic fluid with negative pressure. Its nature is unclear.

In simplest interpretation dark energy is cosmological constant (vacuum energy) ${ }^{4}$ and its density is about $70 \%$ of the total energy density in the Universe. Despite of the agreement with observational data, the $\Lambda$ CDM model is not able to explain some evidences at astrophysical scales. Explaining flat rotation curves in spiral galaxies requires the so called dark matter. But there is disagreement between predictions from N-body simulations for the LCDM model and low density dark haloes observed in some such systems (like low surface brilliance (LSB) galaxies). ${ }^{5}$ Another problem of $\Lambda$ CDM model has deep theoretical roots. One needs to explain the huge difference

This is an Open Access article published by World Scientific Publishing Company. It is distributed under the terms of the Creative Commons Attribution 4.0 (CC-BY) License. Further distribution of this work is permitted, provided the original work is properly cited. 
between observed value of vacuum energy and the one predicted by any quantum gravity (the puzzle of cosmological constant ${ }^{6}$ ).

One may consider these contradictions as indication that General Relativity cannot describe the Universe at cosmological level although it is well tested on Solar System scales. One of the real alternative to dark energy is modified gravity. ${ }^{7-9}$ The early inflation and observed acceleration can be described in the same manner in modified gravity. ${ }^{10-12}$

Of course one needs to verify any modified gravity on all scales. One can expect that modifying the gravitational field action leads to consequences at astrophysical level. Although modified gravity in the weak field limit should reproduce results of General Relativity, the strong field regimes could discriminate between General Relativity and its possible extensions. Strong gravitational fields naturally exist in relativistic compact stars (neutron stars) making these objects very interesting for investigation of possible deviations from General Relativity. One can obtain also some new phenomena in stellar astrophysics which cannot be described by Einstein Gravity. Their possible observational evidences could be in favor of modified gravity. ${ }^{13-14}$

The plan of paper is following. In Section 2 we present the Tolman-OppenheimerVolkoff (TOV) equations for $f(R)$ gravity. In Section 3 we briefly discuss description of dense matter in compact stars and corresponding equation of state (EoS). In Section 4 we consider possible signatures of modified gravity in neutron star astrophysics.

\section{The Tolman-Oppenheimer-Volkoff Equations in $f(R)$ Gravity}

The action for gravitational field and matter is written for $f(R)$ gravity as $(G=1$ and $c=1$ )

$$
S=\frac{1}{16 \pi} \int d^{4} x \sqrt{-g} f(R)+S_{\text {matter }}
$$

where $f(R)$ is some function of scalar curvature $R$. For static and spherically symmetric stars the metric can be written in form

$$
d s^{2}=-e^{2 \phi(r)} d t^{2}+e^{2 \lambda(r)} d r^{2}+r^{2}\left(d \theta^{2}+\sin ^{2} \theta d \phi^{2}\right) .
$$

The two functions $\phi(r)$ and $\lambda(r)$ depend on radial coordinate only. The energymomentum tensor for matter can be taken as

$$
T_{\mu \nu}=\operatorname{diag}\left(e^{2 \phi} \rho c^{2}, e^{2 \lambda} P, r^{2} P, r^{2} \sin ^{2} \theta P\right) .
$$

Here $\rho$ and $P$ are energy density and pressure of the matter, respectively. correspondingly. Variation of action on metric gives the following field equations

$$
\frac{f^{\prime}(R)}{r^{2}} \frac{d}{d r}\left[r\left(1-e^{-2 \lambda}\right)\right]=8 \pi \rho+\frac{1}{2}\left(f^{\prime}(R) R-f(R)\right)
$$




$$
\begin{gathered}
+e^{-2 \lambda}\left[\left(\frac{2}{r}-\frac{d \lambda}{d r}\right) \frac{d f^{\prime}(R)}{d r}+\frac{d^{2} f^{\prime}(R)}{d r^{2}}\right] \\
\frac{f^{\prime}(R)}{r}\left[2 e^{-2 \lambda} \frac{d \psi}{d r}-\frac{1}{r}\left(1-e^{-2 \lambda}\right)\right]=8 \pi p+\frac{1}{2}\left(f^{\prime}(R) R-f(R)\right) \\
+e^{-2 \lambda}\left(\frac{2}{r}+\frac{d \psi}{d r}\right) \frac{d f^{\prime}(R)}{d r} .
\end{gathered}
$$

The hydrostatic equilibrium condition remains the same as in General Relativity:

$$
\nabla^{\mu} T_{\mu \nu}=0 \rightarrow \frac{d p}{d r}=-(\rho+p) \frac{d \psi}{d r}
$$

Finally, from the trace of Einstein equations one obtain the equation for scalar curvature:

$$
\begin{gathered}
3 \square(f(R)-R)+f^{\prime}(R) R-2 f(R)=-8 \pi(\rho-3 p), \\
e^{2 \lambda} \square=\left(\frac{2}{r}+\frac{d \psi}{d r}-\frac{d \lambda}{d r}\right) \frac{d}{d r}+\frac{d^{2}}{d r^{2}} .
\end{gathered}
$$

For a given $\operatorname{EoS} p=f(\rho)$ one can integrate Eqs. (3)-(6) starting from center of star. It is convenient to define gravitational mass $m(r)$ according to the relation ${ }^{15}$

$$
e^{-2 \lambda}=1-\frac{2 m}{r}
$$

Assuming that additional term in Lagrangian is small in comparison with scalar curvature i.e.

$$
f(R)=R+\alpha h(R), \quad \alpha h(R)<<R
$$

one can solve the modified TOV equations by perturbative approach. ${ }^{16-18}$ For a perturbative solution, the density, pressure, mass and curvature can be expanded as

$$
\begin{gathered}
p=p^{(0)}+\alpha p^{(1)}+\ldots, \quad \rho=\rho^{(0)}+\alpha \rho^{(1)}+\ldots, \\
m=m^{(0)}+\alpha m^{(1)}+\ldots, \quad R=R^{(0)}+\alpha R^{(1)}+\ldots
\end{gathered}
$$

The functions $\rho^{(0)}, p^{(0)}, m^{(0)}$ and $R^{(0)}$ satisfy the standard TOV equations. Terms containing $h(R)$ are assumed to be of first order in the small parameter $\alpha$. 
For self-consistent (non-perturbative) solution it is useful to describe the same task in terms of scalar-tensor theory. For $f(R)$ gravity one can construct equivalent Brans-Dicke theory with the action for gravitational sector:

$$
S_{g}=\frac{1}{16 \pi} \int d^{4} x \sqrt{-g}(\Phi R-U(\Phi)) .
$$

Under conformal transformation $\tilde{g}_{\mu \nu}=\Phi g_{\mu \nu}$ the action is rewritten in the Einstein frame

$$
S_{g}=\frac{1}{16 \pi G} \int d^{4} x \sqrt{-\tilde{g}}\left(\tilde{R}-2 \tilde{g}^{\mu \nu} \partial_{\mu} \phi \partial_{\nu} \phi-4 V(\phi)\right),
$$

where $\phi=\frac{\sqrt{3}}{2} \ln \Phi$ and the redefined potential in Einstein frame $V(\phi)$ is $V(\phi)=$ $\Phi^{-2}(\phi) U(\Phi(\phi)) / 4$.

It is convenient to choose space-time metric in the form formally coincident with (2) but with redefined functions $\psi$ and $\lambda$ :

$$
d \tilde{s}^{2}=\Phi d s^{2}=-e^{2 \tilde{\psi}} d t^{2}+e^{2 \tilde{\phi}} \tilde{d r}^{2}+\tilde{r}^{2} d \Omega^{2} .
$$

The resulting equations for metric functions $\tilde{\lambda}$ and $\tilde{\psi}$ coincide in fact with ordinary TOV equations in which energy density and pressure of scalar field $\phi$ are included (tildes are omitted for simplicity):

$$
\begin{gathered}
\frac{1}{r^{2}} \frac{d}{d r}\left[r\left(1-e^{-2 \lambda}\right)\right]=8 \pi e^{-4 \phi / \sqrt{3}} \rho+e^{-2 \lambda}\left(\frac{d \phi}{d r}\right)^{2}+V(\phi), \\
\frac{1}{r}\left[2 e^{-2 \lambda} \frac{d \psi}{d r}-\frac{1}{r}\left(1-e^{-2 \lambda}\right)\right]=8 \pi e^{-4 \phi / \sqrt{3}} p+e^{-2 \lambda}\left(\frac{d \phi}{d r}\right)^{2}-V(\phi),
\end{gathered}
$$

The hydrostatic equilibrium condition can be rewritten as

$$
\frac{d p}{d r}=-(\rho+p)\left(\frac{d \psi}{d r}-\frac{1}{\sqrt{3}} \frac{d \phi}{d r}\right)
$$

Finally, the last equation of motion for scalar field is equivalent to Eq. (6) in $f(R)$ theory:

$$
\square \phi+\frac{d V(\phi)}{d \phi}=-\frac{4 \pi}{\sqrt{3}} e^{-4 \phi / \sqrt{3}}(\rho-3 p) .
$$

\section{Equation of State for Nuclear Matter}

Let us use a simple model for describing nuclear matter in neutron stars. For nuclear matter consisting of baryon octet $\left(b=p, n, \Lambda, \Sigma^{0, \pm}, \Xi^{0,-}\right)$ and leptons $\left(l=e^{-}, \mu^{-}\right)$ 
interacting with magnetic field and scalar $\sigma$, isoscalar-vector $\omega_{\mu}$ and isovector-vector $\rho_{\mu}$ meson fields and leptons the Lagrangian is ${ }^{19,20}$

$$
\begin{gathered}
\mathcal{L}=\sum_{b} \bar{\psi}_{b}\left(\gamma_{\mu}\left(i \partial^{\mu}-q_{b} A^{\mu}-g_{\omega b} \omega^{\mu}-\frac{1}{2} g_{\rho b} \tau \cdot \rho^{\mu}\right)-\left(m_{b}-g_{\sigma b} \sigma\right)\right) \psi_{b} \\
+\sum_{l} \bar{\psi}_{l}\left(\gamma_{\mu}\left(i \partial^{\mu}-q_{l} A^{\mu}\right)-m_{l}\right) \psi_{l}
\end{gathered}
$$

$+\frac{1}{2}\left(\left(\partial_{\mu} \sigma\right)^{2}-m_{\sigma}^{2} \sigma^{2}\right)-V(\sigma)-\frac{1}{4} F_{\mu \nu} F^{\mu \nu}+\frac{1}{2} m_{\omega}^{2} \omega^{2}-\frac{1}{4} \omega_{\mu \nu} \omega^{\mu \nu}-\frac{1}{4} \rho_{\mu \nu} \rho^{\mu \nu}+\frac{1}{2} m_{\rho}^{2} \rho_{\mu}^{2}$.

Solving equations for meson and fermion fields one can obtain the dependence $p(\rho)$ (for details of calculations see Refs. 21-23). For hyperon-meson couplings and other parameters of the model see Ref. 20 (TW model) and Ref. 19 (GM model).

For magnetic field one assume the parametrization proposed in Ref. 25:

$$
B=B_{s}+B_{0}\left(1-\exp \left(-\gamma_{2}\left(n / n_{s}\right)^{\gamma_{1}}\right)\right) .
$$

$B_{s} \sim 10^{15} \mathrm{G}$ is magnetic field on surface, $B_{0}=B_{c} B_{0}^{*}$ where $B_{c}=4.414 \times 10^{13} \mathrm{G}$ is relativistic field for electrons. The parameters $\gamma_{1}$ and $\gamma_{2}$ define the rapidity and sharpness of increasing magnetic field with increasing baryon density $n$.

\section{Possible Signatures of Modified Gravity}

The recent discovery of the pulsars PSR J1614-223026 and PSR J0348+0432 27 excluded various matter EoS for neutron stars at high densities, including hyperons and/or quarks EoS. But according to realistic models for strong interactions, the hyperons appear at densities $5-8 \times 10^{14} \mathrm{~g} / \mathrm{cm}^{3}$. This softens the EoS and reduces maximal mass. One can search the solution of hyperon puzzle within particle physics or in frames of modified gravity.

In a simple model with $f(R)=R+\beta R^{3}$ it is possible to construct neutron star models with $M \sim 2 M_{\odot}$ for simple EoS from GM2 and GM3 model extended to hyperon sector. For example, we found that for gm2nph and gm3nph EoS (for analytical approximation of these EoS see Ref. 28) the two solar mass limit can be achieved for $\beta=-40$ and $\beta=-45$ (in units of $r_{0}^{4}=G^{4} M_{\odot}^{4} / c^{8}$ ), respectively (see Fig. 1). But in General Relativity the maximal masses of stars for these EoS are $1.66 M_{\odot}(\mathrm{gm} 2 \mathrm{nph})$ and $1.57 M_{\odot}(\mathrm{gm} 3 \mathrm{nph})$.

For more realistic model with

$$
f(R)=R+\alpha R^{2}+\beta R^{3}
$$

the increase of maximal neutron star mass takes place also at $\beta<0$ if the cubic term is larger than the quadratic one at high densities. The mass-radius relation is close to that for the gravity without a quadratic term.

Another interesting feature of mass-radius relation in modified gravity is the existence of second branch of stable stars with high central densities (and strong magnetic fields in the case of magnetars). For strong magnetic fields the mass-radius 
Table 1. The parameters of neutron stars on the second branch of stability for TW EoS and R-squared gravity $\left(\gamma_{1}=3, \gamma_{2}=0.02\right)$. The density and magnetic field in center are given for star configuration with maximal mass.

\begin{tabular}{cccccc}
\hline$B_{0}$, & $\alpha$, & $M_{\max }$, \\
$10^{5}$ & $r_{0}^{2}$ & $\begin{array}{c}R, \\
\mathrm{~km}\end{array}$ & $\begin{array}{c}\rho_{c}, \\
10^{15} \mathrm{~g} / \mathrm{cm}^{3}\end{array}$ & $\begin{array}{c}B_{c}, \\
10^{18} \mathrm{G}\end{array}$ \\
\hline 2 & 0.5 & 2.29 & 11.45 & 2.64 & 5.96 \\
\hline 3 & 0.5 & 2.55 & 12.45 & 2.50 & 6.07 \\
\hline
\end{tabular}

relation considerably changes from that in General Relativity only for masses close to maximal. For example for $\gamma_{1}=3, \gamma_{2}=0.02$ in model (17) the second branch of stable stars (for which $d M / d \rho_{c}>0$ ) may appear for high central densities (see Fig. 2 and Table 1).

\subsection{Nonperturbative models of compact stars in $f(R)$ gravity}

In frames of perturbative approach one assume a Schwarzschild solution outside the star. For nonperturbative solution one needs to solve TOV equations with $\rho=p=0$. One needs also to impose the junction conditions on the surface of the star $\left(r=r_{s}\right)$ :

$$
\lambda_{\text {in }}\left(r_{s}\right)=\lambda_{\text {out }}\left(r_{s}\right), \quad R_{\text {in }}\left(r_{s}\right)=R_{\text {out }}\left(r_{s}\right), \quad R_{\text {in }}^{\prime}\left(r_{s}\right)=R_{\text {out }}^{\prime}\left(r_{s}\right)
$$

and the asymptotic flatness requirement:

$$
\lim _{r \rightarrow \infty} R(r)=0, \lim _{r \rightarrow \infty} m(r)=\text { const. }
$$

The consideration for simple quark $\operatorname{EoS} p=c(\rho-4 B)$ shows the following (see Fig. 3 and Table 2). For a given central density there is one value of scalar curvature at which stable star exist (in $R^{2}$-gravity). ${ }^{29,30}$ In the corresponding scalar-tensor

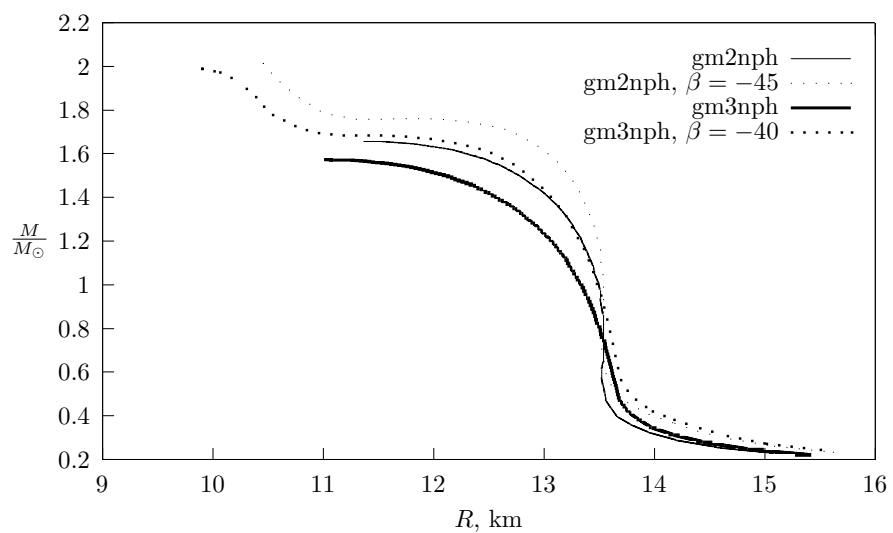

Fig. 1. Mass-Radius diagram for gm2nph and gm3nph EoS in modified gravity $f(R)=R+\beta R^{3}$ (dots) with maximal mass $\sim 2 M_{\odot}$ for some values of $\beta$. For comparison, the mass-radius relation for General Relativity is given by solid lines for these EoS. 


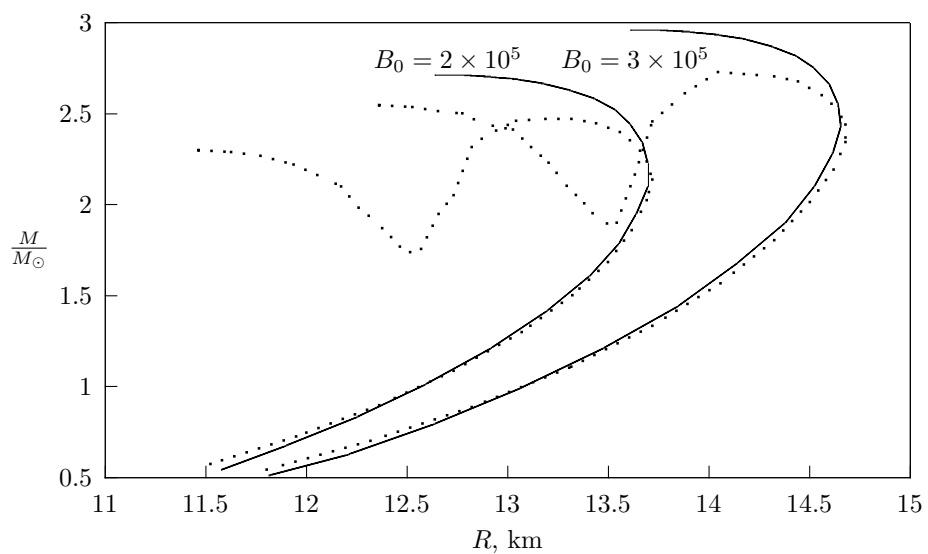

Fig. 2. Mass-radius diagram for TW EoS in R-squared gravity (dotted lines) in comparison with General Relativity (solid lines) for $\alpha=0.5$ (in units of $r_{0}^{2}$ ) in a case of $B_{0}=2 \times 10^{5}$ and $3 \times 10^{5}$. The second branch of stability appears.

description the fine tuning for scalar field is needed. Increasing $\alpha$ leads to growth of gravitational mass for distant observer. But the interpretation of this increase 

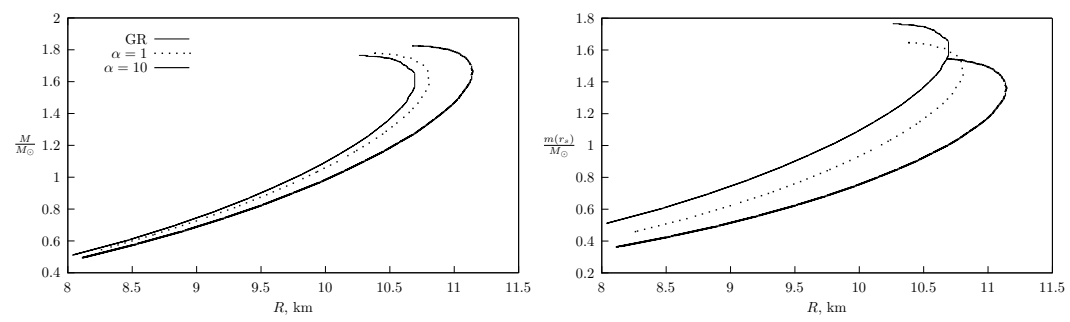

Fig. 3. The mass-radius diagram (left panel) in model $f(R)=R+\alpha R^{2}$ and in GR for quark stars with EoS $p=c(\rho-4 B)\left(B=60 \mathrm{MeV} / \mathrm{fm}^{3}, c=0.28\right)$. On right panel the dependence of stellar mass $m\left(r_{s}\right)$ on radius in Jordan frame is depicted.

Table 2. Quark star properties for simple EoS $p=c(\rho-4 B)$ with $c=0.28$ and $B=60$ $\mathrm{MeV} / \mathrm{fm}^{4}$ in $R$-squared gravity.

\begin{tabular}{ccccc}
\hline$\alpha$, & $M_{\max }$, & $m\left(r_{s}\right)$, & $r_{s}$, & $\rho_{c}$, \\
$r_{0}^{2}$ & $M_{\odot}$ & $M_{\odot}$ & $\mathrm{km}$ & $10^{15} \mathrm{~g} / \mathrm{cm}^{3}$ \\
\hline 0 & 1.764 & 1.764 & 10.26 & 2.17 \\
1 & 1.778 & 1.649 & 10.38 & 2.26 \\
10 & 1.832 & 1.552 & 10.68 & 2.54 \\
\hline
\end{tabular}

depends on the framework. In $f(R)$ theory one can say that the increase of mass occurs by "gravitational sphere" outside the star with some "effective mass". The mass $m\left(r_{s}\right)$, however, decreases with increasing $\alpha$ (as in the perturbative approach). In scalar-tensor theory for conformal frame we also have the so called dilaton sphere outside the star but its contribution into gravitational mass for a distant observer is negligible.

\section{Conclusion}

Modified gravity may be consistent with current constraints on the maximum neutron star mass limit and on the data on neutron star masses and radii, and it may simplify the solution of hyperon problem in superdense matter.

For large densities and/or magnetic fields, considerable effects can be induced on EoS and therefore the modified gravity effects can appear. Other branches of neutron stars are possible considering the extra pressure contributions coming from gravity extensions. If such stars will be explicitly observed, this could be considered as a clear signature in favor of modified gravity.

\section{Acknowledgments}

This work is supported by project \# 14-01-31100 (RFBR, Russia) and project \# 2058 (MES, Russia). 


\section{References}

1. S. Perlmutter et al. [Supernova Cosmology Project Collaboration], Astrophys. J. 517, 565 (1999).

2. A. G. Riess et al. [Supernova Search Team Collaboration], Astron. J. 116, 1009 (1998).

3. A. G. Riess et al. [Supernova Search Team Collaboration], Astrophys. J. 607, 665 (2004).

4. N. A. Bahcall et al., Science 284, 1481 (1999).

5. W. J. G. de Blok, S. S. McGaugh and A. Bosnia, Mon. Not. Roy. Astron. Soc. 340, 657 (2003).

6. S. Weinberg, Rev. Mod. Phys. 61, 1 (1989).

7. S. Capozziello, Int. J. Mod. Phys. D 11, 483 (2002).

8. S. Capozziello, S. Carloni and A. Troisi, Recent Res. Dev. Astron. Astrophys. 1, 625 (2003).

9. S. Nojiri and S. D. Odintsov, Phys. Rev. D 68, 123512 (2003).

10. S. Nojiri and S. D. Odintsov, Phys. Rept. 505, 59 (2011).

11. S. Nojiri and S. D. Odintsov, Phys. Lett. B 576, 5 (2003).

12. S. Capozziello and M. De Laurentis, Phys. Rept. 509, 167 (2011).

13. S. Capozziello, M. De Laurentis, I. De Martino, M. Formisano and S. D. Odintsov, Phys. Rev. D 85, 044022 (2012).

14. S. Capozziello, M. De Laurentis, S. D. Odintsov and A. Stabile, Phys. Rev. D 83, 064004 (2011).

15. H. Stephani, General Relativity (Cambridge Univ. Press, Cambridge, 1990).

16. S. Arapoglu, C. Deliduman and K. Y. Ekşi, JCAP 1107, 020 (2011).

17. H. Alavirad and J. M. Weller, Phys. Rev. D 88, 124034 (2013).

18. A. Astashenok, S. Capozziello and S. Odintsov, JCAP 12, 040 (2013).

19. N. K. Glendenning and S. A. Moszkowski, Phys. Rev. Lett. 67, 2414 (1991).

20. S. Typel and H. H. Wolter, Nucl. Phys. A 656, 331 (1999).

21. A. Broderick, M. Prakash and J. M. Lattimer, Astrophys. J. 537, 351 (2000).

22. A. Broderick, M. Prakash and J. M. Lattimer, Phys. Lett. B 531, 167 (2002).

23. C. Y. Cardall, M. Prakash and J. M. Lattimer, Astrophys. J. 554, 322 (2001).

24. C.-Y. Ryu et al., Phys. Rev. C 85, 045803 (2012).

25. C.-Y. Ryu, K. S. Kim and M. K. Cheoun, Phys. Rev. C 82, 025804 (2010).

26. P. B. Demorest et al., Nature 467, 1081 (2010).

27. J. Antoniadis, P. C. C. Freire, N. Wex et al., Science 340, 448 (2013).

28. C. Güngör and K. Y. Ekşi, Analytical representation for equations of state of dense matter, arXiv:1108.2166.

29. K. V. Staykov, D. D. Doneva, S. S. Yazadjiev and K. D. Kokkotas, JCAP 1406, 003 (2014).

30. A. V. Astashenok, S. Capozziello and S. D. Odintsov, Phys. Lett. B 742, 160 (2015). 